\title{
A Model for Horizontal Line Array Deployment
}

\author{
David S. Hammond \\ Hydromechanical Branch Code 5043 \\ Naval Air Warfare Center \\ Aircraft Division, Warminster
}

\author{
Dr. James F. McEachern \\ Advanced Concepts Branch Code 5044 \\ Naval Air Warfare Center \\ Aircraft Division, Warminster
}

\begin{abstract}
The in-water deployment of large cable systems onthe-bottom in a prescribed manner is a problem found in many applications. This paper describes an algorithm developed to dynamically model the deployment of large horizontal cable systems from the surface to the ocean floor and discusses experimental validation techniques. The validated model can be used as a design tool to facilitate the deployment of long cable systems without the expense of building experimental mock-ups.
\end{abstract}

\section{INTRODUCTION}

The technique of laying a string of cable connected sensors on the surface of the ocean and executing a controlled free fall to an orderly landing on the bottom is being developed. If the string is an array of acoustic sensors, the final position of the sensors is critical to the operation of the system.

The array is required to descend through the water column and lay on the bottom in a straight line. For maximum utility, this scenario must take place regardless of array orientation, current profile, or sea state; therefore, a reliable, robust technique must be developed to situate the array of sensors on the bottom.

In general, a two pronged approach is being taken to accomplish stable and consistent deployment of horizontal line arrays. A computer model is being developed to serve as a design tool to evaluate various deployment schemes. The candidate techniques will be further investigated through scale model experimentation, and a full scale, proof of concept, array will be built utilizing the knowledge gained during this effort.

\section{HORIZONTAL LINE ARRAY DEPLOYMENT (HLAD) MODEL}

The purpose of the HLAD computer model is to act as a design tool in the development of deployment techniques for extended line sensor arrays. This allows the designer the option of evaluating many deployment alternatives with minimal development cost.

\section{A. Theory}

The HLAD model is based on well established theory documented by several authors for various hydrodynamic cable models $[1,2,3]$. Due to the uniqueness of extended line array deployment, it was decided that a new model needed to be developed based on previous cable model theory, so the specific issues involved with extended sensor arrays could be better addressed.

The system under consideration consists of three major elements: cables (signal cable, fiber optic cable), bodies (sensors, lifting bodies, anchors), and environmental conditions (density, currents, ocean depth). A typical simulation may consist of some or all of these elements, but the overall system length is often the driving factor, especially when the lengths are on the magnitude of miles.

Because of the large scale of the proposed systems, some general assumptions were made to keep the problem manageable. The system is initially positioned horizontally on the surface. There is no tension in the cable and the system is at rest. Relatively little time is spent on the surface when compared to in water descent time, so it was assumed that the wave induced motions could be neglected. Variations in current direction were also neglected in order to keep the system in two dimensions. Fig. 1 shows the global coordinate system, labeled $\mathrm{x}$ and $y$, as positive $x$ to the right and positive $y$ downward. Angle $\phi$ is measured from the positive $y$ axis in a clockwise positive manner. Finally, for ease of computation the cable segments were assumed to be linear but extensible.

Each cable is defined by its physical properties: length, diameter, wet weight per foot, mass per foot, normal and tangential drag coefficients, and elastic modulus. Cable orientation and motions are determined by the forces that act on the cable. Forces acting on the cable are due to gravity, inertia, drag, and tension. Fig. 1 depicts these forces along with the local cable coordinate system, $c_{n}$ (cable normai) and $c_{t}$ (cable tangent).

Gravity forces are distributed along the length of the cable in the positive global $y$ direction. No transformations are necessary and the total force is equal to the length of the cable times the wet weight of the cable per foot. 
Inertial forces act in the direction of the cable acceleration. Added mass in the cable is considered negligible; therefore inertial forces are equal to the acceleration in the $x$ or $y$ directions times the mass of the cable.

The drag forces experienced by the cable are distributed along the cable in the normal and tangential directions. These forces are governed by the equation,

$$
F_{d}=\frac{1}{2} \rho C d A \mathbf{V}^{2}
$$

where

$F_{d}$ is the force due to drag

$\rho$ is the density of the water

$\mathrm{Cd} A$ is the drag area of the cable

$\mathrm{V}$ is the velocity of the cable through the fluid.

The normal and tangential drag forces are in opposition to the normal and tangential velocities. The relative velocity of the cable, including the current, is transformed into normal and tangential cable coordinates and the normal and tangential drags are calculated. These drag forces are then transformed into the global $x$ and $y$ coordinates for use in the equations of motion.

The typical Reynolds numbers experienced by the proposed systems are such that strumming can occur in some cables; therefore a strum model has been included in the drag calculations of the cables. The strum frequency of a particular cable is defined [4] by:

$$
f_{s}=\frac{s t \cdot v}{d}
$$

where

St is the Strouhal number

$V$ is the velocity normal to the cable

$d$ is the diameter of the cable.

When the strum frequency approaches the natural frequency of the cable, it is said to be locked on and the drag increases. The increase in drag can be shown to be [4]:

$$
\frac{C d s}{C d}=1+1.699\left\{\frac{1}{(1+4.6 / \sqrt{\mathrm{Re}})^{2.1775}}\right\}
$$

where strumming

$\mathrm{C}_{\mathrm{ds}}$ the average drag coefficient due to

$C_{d}$ is the normal cable drag coefficient

$\mathrm{Re}$ is the Reynolds number.

If the strum frequency is within two percent of the cable natural frequency, the normal drag coefficient is adjusted using the above relationship.

Cable tension is a function of the cable strain and strain rate defined by the following equation:

$$
T=T_{o}+A E \varepsilon+C_{d a m p} \dot{\varepsilon}
$$

where

$T$ is the cable tension

$T o$ is the cable reference tension

$A E$ is the elastic modulus times cross sectional

area

$$
\begin{aligned}
& \varepsilon \text { is the cable strain }=\left(L-L_{o}\right) / L_{o} \\
& C_{d a m p} \text { is the inertial damping coefficient } \\
& \dot{\varepsilon} \text { is the strain rate } \\
& L_{o} \text { is the cable segment reference length. }
\end{aligned}
$$

Cable tension acts in the $c_{t}$ axis direction and is transformed into the $x$ and $y$ global coordinates for use in the equations of motion.

In typical systems considered in this application, bodies refer to acoustic sensors, electronic housings, drag devices such as parachutes or drogues, lifting devices, and anchors or ballast. Bodies lie at the ends of cable segments and are defined by their physical properties: wet weight, lift and drag characteristics, and virtual mass (mass plus added mass) in the $x$ and $y$ directions. The forces that act on the bodies are inertial, gravitational, drag and lift, and the forces due to the attached cables seen as tension above the body (Ta) and tension below the body (Tb). In a similar fashion as the cables, the motions and orientations of the bodies are functions of the forces on the bodies.

Lift and drag properties are calculated in the velocity coordinate system. In fig. 2, the velocity coordinate system consists of $V_{n}$ in the direction of the relative velocity and $V_{t}$ orthogonal to $V_{n}$. These are then transformed into the local body coordinate system, $B_{n}$ and $B_{t}$, then into the global $x$ and $y$ coordinate systems with the appropriate transformation matrix. The body angle of attack, $\alpha$, is measured from the $V_{n}$ axis to the $B_{t}$ axis.

The environmental characteristics affect the motions and final position of the system to a high degree. The current profile and density of the water, drive the drag induced forces, while depth of the bottom influences descent time and sensor final positioning. In the future an air-water interface will be incorporated so that the descent of the system can be tracked from splash down to the ocean floor.

The equations of motion are expressed in the $x$ and $y$ components. They are, in a generalized form, as follows:

$$
\begin{aligned}
& {\left[\mathbf{M x}_{\mathbf{x}}\right] \cdot \overrightarrow{\mathbf{A}}_{\mathbf{x}}=\overrightarrow{\mathbf{F}}_{\mathbf{x}}} \\
& {\left[\mathbf{M}_{\mathbf{y}}\right] \cdot \overrightarrow{\mathbf{A}}_{\mathbf{y}}=\overrightarrow{\mathbf{F}}_{\mathbf{y}}}
\end{aligned}
$$

where 
$\left[\mathbf{M}_{\mathbf{x}}\right]$ is an $\mathbf{N} \times \mathbf{N}$ matrix $=\left[\mathbf{M}_{\mathbf{c a b}}\right]+\left[\mathbf{M}_{\mathbf{v}, \text { body, }}\right]$ $\left[\mathbf{M}_{\mathbf{y}}\right]$ is an $\mathrm{N}$ x N matrix $=\left[\mathbf{M}_{\text {cab }}\right]+\left[\mathbf{M}_{\mathbf{v}}\right.$,body,y $]$ $\mathrm{N}$ is the number of cables in the system

$\left[\mathbf{M}_{\mathbf{v}, \text { body, }}\right]$ is the body virtual mass in the $\mathrm{x}$ direction

$\left[\mathbf{M}_{\mathbf{v}}\right.$, body, $\left.y\right]$ is the body virtual mass in the $y$ direction

$\overrightarrow{\mathbf{A}_{x}}$ is the $\mathbf{x}$ acceleration vector

$\vec{A}_{\mathbf{y}}$ is the $y$ acceleration vector

$\overrightarrow{\mathbf{F}}_{\mathbf{x}}$ is the $\mathbf{x}$ direction forces vector

$\vec{F}_{y}$ is the y direction forces vector.

Expanding the force vectors:

$\overrightarrow{\mathbf{F}}_{\mathbf{x}}=[\mathbf{C}] \overrightarrow{\mathbf{T}}_{\mathbf{x}}+[\mathbf{C}]\left(\mathbf{D}_{\mathbf{n}}+\mathbf{D}_{\mathbf{t}}\right)_{x}+[\mathbf{B}](\mathbf{D}+\mathbf{L})_{x}$

$\overline{\mathbf{F}}_{\mathbf{y}}=[\mathrm{C}] \overline{\mathbf{T}}_{\mathbf{y}}+[\mathrm{C}]\left(\mathbf{D}_{\mathbf{n}}+\mathbf{D}_{\mathbf{t}}\right)_{y}+[\mathrm{B}](\mathrm{D}+\mathbf{L})_{y}+\mathbf{W}_{\mathbf{c a b}}+\mathbf{W}_{\mathbf{b o d}}$

where

$[C]$ is the cable transformation matrix

$[\mathrm{B}]$ is the body transformation matrix

$\overrightarrow{\mathbf{T}}$ is the tension vector

$D_{n}$ and $D_{t}$ are normal and tangential drag vectors

$\mathbf{D}$ and $\mathbf{L}$ are the body drag and lift vectors

$W_{\text {cab }}$ and $W_{\text {bod }}$ are the cable and body weight vectors.

The velocity and position vectors are determined

by integrating the acceleration vectors to obtain the classical equations for velocity and position.

\section{B. Computer Model}

The HLAD model was developed in response to the unique situation presented by deploying extended line arrays. Using the theory outlined above, it is capable of dynamically simulating the deployment of long line sensor arrays in any two dimensional current profile, and with variable lift and drag bodies.

Input menus receive data for general information, cable properties, body properties, and environmental characteristics. This information is written to an input file that can be edited independent of the program. The input file provides all the information for the main HLAD model to function. All of the model inputs are similar to those found in conventional cable models $[1,2,3]$ with the exception of the body lift and drag computations.

Accurate models for body lift and drag are vital to the success of the HLAD computer model; therefore extra emphasis has been placed in the development of this capability. Flexibility in the characterization of the bodies was a priority. There are three ways to input lift and drag information to the program. For bodies in which lift and drag play a reduced role, acoustic sensor for example, constant coefficients for normal drag, tangential drag and lift can be entered. If a device was tested experimentally, and lift and drag forces are available for different angles of attack and velocities, this information can be input in a tabular form. The model performs a double interpolation to calculate drag and lift forces for a particular orientation and relative velocity. Finally if experimental data or theoretical computations produce an equation for lift and drag as a function of angle of attack and relative velocity, such as those found in Hoerner [5,6], the equations can be input directly into the model.

A lumped node parameter method is used in the HLAD model. The forces and equations of motion are applied at nodes along the cable in incremental time steps until the system reaches the bottom. Half of the individual cable forces are applied at a node above the cable and half are applied at a node below the cable. The number of nodes is user selected, and the length of the time steps is automatically adjusted by the program to insure convergence with minimum runtime. The cable sections, or elements between the nodes are considered linear, massless and rigid. At time zero, the system is in a horizontal orientation with no tension and at rest. The weights at the nodes force the system into motion in the positive $y$ direction. At this initial stage, weight is the only force acting on the nodes. Solution for accelerations and the resulting positions and velocities is trivial. As time is incremented, drag, tension and inertial effects become important. These forces are summed at the nodes in the $x$ and $y$ directions and the nodal accelerations are calculated. Using a forward finite difference approach, the velocities and positions are calculated. The simulation time is incremented by the time step and the new positions and velocities are used to calculate a new set of nodal forces. This process continues until a node reaches the user defined bottom at which point the position of the node is fixed.

The solution technique outlined above is admittedly crude when compared to the sophisticated methods available today [7]; however, the computational cost of using a more accurate method is prohibitive for the systems modeled by HLAD, specifically very long arrays. This method was chosen for its simplicity and ease of computation, and it will allow for first cut designs to be evaluated in a general but reliable and physically valid way. HLAD is undergoing intensive validation before it can be used for full scale modeling in a confident manner. Additionally, the model can be easily updated to use nonlinear elements or use a more accurate solution technique because the basis of the program, calculation of the nodal forces, will not change.

The HLAD model provides the user with output data in two forms. A print file is produced that gives information on nodal positions, velocities, cable angle, tension and stretched length, as well as lift and drag data for the bodies. The output is written to a file at user 
specified intervals and a hard copy is produced through the main program. A plot file is also produced, giving nodal position data at the same intervals as the print file. 'A commercially available plotting spreadsheet software can be used to generate time lapse plots of the descending array.

\section{END BODY PARAMETRIC DESIGN STUDY}

A design study was undertaken using the HLAD computer model to investigate the effect on deployment of varying end body properties. The basic array consisted of three inch spherical steel elements at ten foot spacing strung together by nylon fishing line. The number of elements, thus array length, was varied. The end bodies were chosen to be flat disks of various sizes and weights, and the depth for all simulations was 40 feet.

Over 100 simulations were run with results that were grouped into four general categories: outer collapse, inner collapse, steady state, and a miscellaneous failure. The parameters that defined the stability of the deployments were the ratio of the total array weight to the end body weight, the weight ratio (WR), and the ratio of the end body drag area to the total array drag area, the drag ratio (DR).

$$
\begin{aligned}
& W R=\frac{\text { Array Weight }}{\text { End Body Weight }} \\
& D R=\frac{\text { End Body Drag Area }}{\text { Array Drag Area }}
\end{aligned}
$$

An outer collapse is characterized by end bodies that are relatively heavy with a small drag area (low WR and DR). These type of deployments tend to have very little tension in the array and the elements generally arrive on-the-bottom very quickly with little spacing.

In an inner collapse deployment, the end bodies are too light and have too little drag (high WR, low DR). This allows the innermost elements of the array achieve a much higher speed than the end bodies. As a result, the center elements reach the bottom far before the outermost, causing the end bodies, with too little drag to resist, to move towards the center. In the end, the array collapses in on itself.

A steady state deployment is the goal of a well designed horizontal array. The weight and drag ratios of steady state type deployments range between the inner and outer collapse regions. All steady state deployments reach a point in its' descent where the velocities of all elements are the same. This stable descent characteristic leads to on-the-bottom element spacing that are the same as they were at the surface.

When the simulations could not be completed due to computational errors, the deployment was put in the miscellaneous failure category. These failures were primarily due to tension that was too high, because of an end body with a great deal of drag, or too low tension due to the opposite condition.

Fig. 3 shows the relationship between the weight and drag ratios and the four deployment groupings. This graph indicates that there is a defined region where the arrays reach a steady state deployment condition, based on the ratios between the end bodies and array weight and drag. This preliminary result does not take the effects of current into account; however it does allow the designer to design a first cut array that will, with some confidence, deploy in a stable manner.

\section{SCALE MODEL TESTING}

Scale model testing is an integral and crucial part of this effort. The testing will serve to gather data for validation of the computer model. Two of the scale model tests are described below and a larger scale open water test will take place in the near future. One of the issues involved with open water tests is the tracking of the array deployment. Visual confirmation of the deployment is not feasible; therefore a tracking technique is under development that will allow the motions of nodes on an array to be recorded directly through the use of miniature data loggers and pressure transducers. This will also serve as additional validation for HLAD.

The initial HLAD validation tests were conducted at the NAWC ten foot tank. Two spherical end weights of various sizes and weights were connected by ten feet of monofilament line of various diameters. When a switch is thrown a solenoid is activated and a signal is sent to trigger a signal analyzer waiting in time capture mode. The action of the solenoid rotates a cradle, dropping the two body array into the water. A hydrophone positioned near the bottom reports to the analyzer when the bodies strike the bottom. Visual conformation is made as to which body strikes the bottom first. A grid placed on the bottom allows the measurement of how far each body traveled from its initial position. This time and position data is compared with results from an identical simulation run on HLAD. Typical results are shown in fig. 4. Fairly good agreement can be seen between the scattered experimental results and the model predictions.

The second tank validation test is to take place at the 100 foot tank at NSWC. Two base arrays will be used in this test. The first array is a $1 / 20^{\text {th }}$ scale representation of a 1000 foot array. This array will be dropped at various depths and with different end bodies. The deployments will be timed and filmed, and the results will be compared to HLAD simulations of each drop. The second array to be tested will be a five element array of autonomous depth logger (ADL) nodes. The ADL nodes consist of a pressure transducer, some conditioning 
electronics and a data logger. The node will record depth versus time data at preset intervals. This test will determine the effectiveness of the ADL nodes and serve as an additional validation for HLAD.

An open water test is scheduled to take place in mid-summer of 1993. This test will use a ten element 1000 foot array of ADL nodes with large parachute type end bodies. The depth history information from the ADL nodes will be augmented by ROV video and acoustic onthe-bottom co-location data. The design of the array, specifically end body drag area and weight was driven by predictions made with HLAD in the parametric end body study described above.

\section{CONCLUSIONS}

The development of a reliable way to deploy inwater on- the bottom extended line sensor arrays is critical to both Navy projects and commercial applications. The cost of this development can be reduced considerably by using the approach taken here. Computer simulations of extended line array deployment allow the designer to evaluate various deployment alternatives without building and testing experimental mockups. Once the design is narrowed down to an optimum solution(s), a mockup can be tested and the deployment can be confirmed using the tracking technique mentioned above. This becomes an iterative process with the designer making refinements to the computer model based on experimental results and retesting the resulting design. Overall, the development cycle for this critical technology becomes much more efficient and the resulting design is better.

\section{REFERENCES}

[1] H.T Wang., "A FORTRAN IV Computer Program for the Time Domain Analysis of the Two Dimensional Dynamic Motions of General Buoy-CableBody Systems," NSRDC Report 77-0046, Jun 1977.

[2] R.L Webster, "SeaDyn Mathematical Models," NCEL Report CR 82.019, Apr 1982.

[3] H.T. Wang, "Description of Computer Program CAB3DYN for the Time Domain Analysis of Three-Dimensional Long-Time Deployment Behavior of General
Ocean Systems," NSRDC Report SPD-0633-03, Dec 1981. [4] R.A. Holler, "Drag Measurements of Long Cables in the Ocean," NAVAIRDEVCEN Report NADC84086-30, Mar 1984.

[5] S.F. Hoerner, Fluid Dynamic Lifi, Liselotte Hoerner, 1975, pp 8.1-17.

[6] S.F.Hoerner, Fluid Dynamic Drag, Sighard Hoerner, 1958, pp 10.1-16.

[7] R.W. Hombeck, Numerical Methods, PrenticeHall Inc, Englewood Cliffs, NJ, pp 185-208.

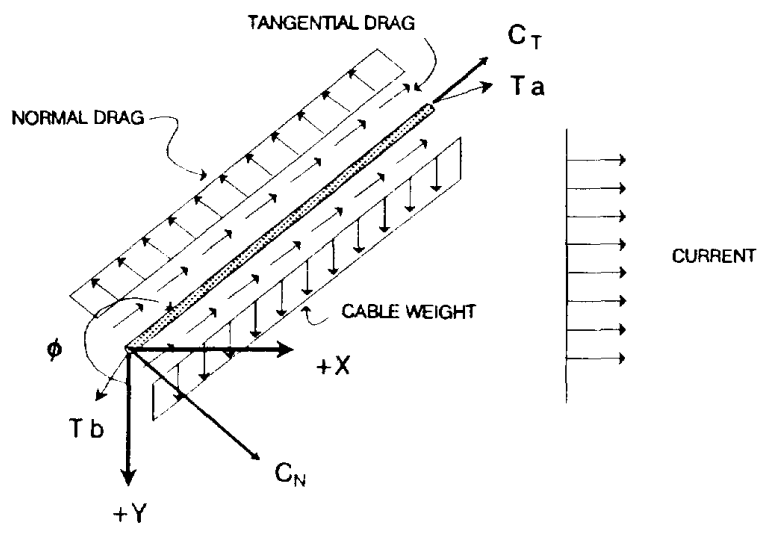

Fig 1. Global and cable coordinate systems.

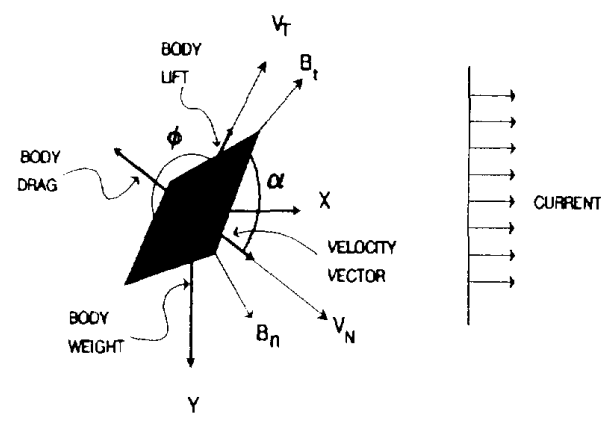

Fig. 2. Body coordinate system. 


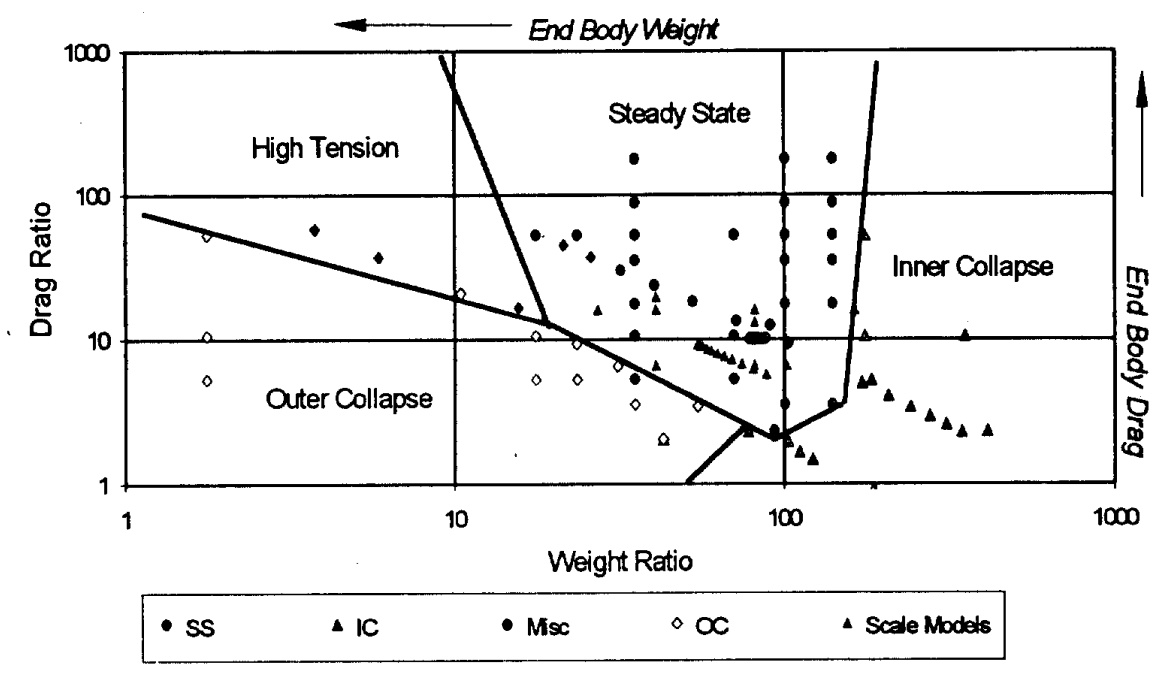

Fig. 3. End body parametric design study results.

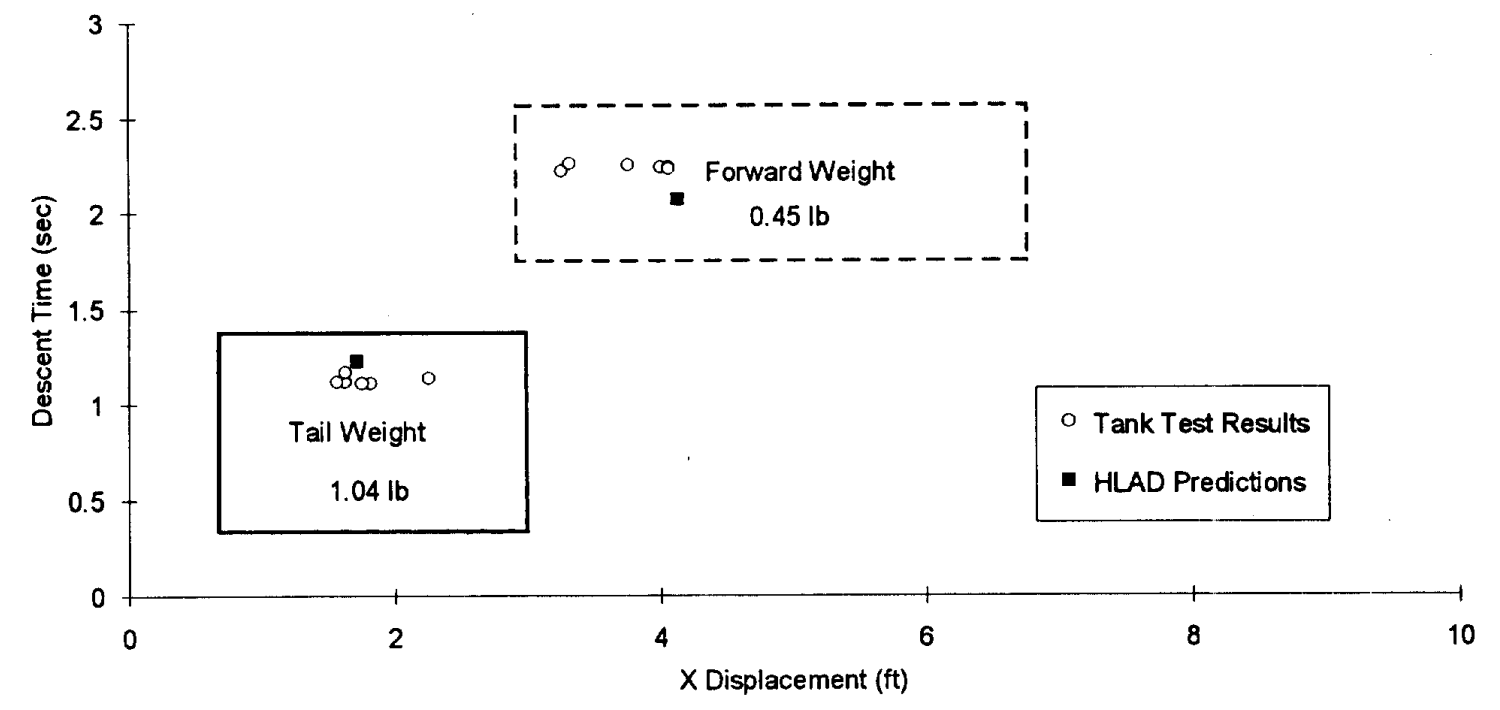

Fig. 4. NAWC ten foot tank validation results. 